\title{
Two-component desorption from anisotropic pore networks
}

\author{
Peter Bräuer \\ Universität Leipzig, Fakultät für Physik und Geowissenschaften, Linnestrasse 5, D-04103 Leipzig, Germany \\ Andreas Brzank ${ }^{a)}$ \\ Institut für Festkörperforschung, Forschungszentrum Jülich, D-52425 Jülich, Germany and Universität \\ Leipzig, Fakultät für Physik und Geowissenschaften, Linnestrasse 5, D-04103 Leipzig, Germany \\ Jörg Kärger \\ Universität Leipzig, Fakultät für Physik und Geowissenschaften, Linnestrasse 5, D-04103 Leipzig, Germany
}

(Received 23 August 2005; accepted 1 December 2005; published online 20 January 2006)

\begin{abstract}
Dynamic Monte Carlo simulations are performed to explore molecular desorption of a two-component mixture from a mode adsorbent of adsorbate-related anisotropy. The intrinsic dynamics of the adsorbent-adsorbate host-guest system is described by assuming a situation typical of a "molecular-traffic-control" system, where the hopping rates of the two components in two directions, perpendicular to each other, are identical, while for each component, perpendicular to that direction of preferential propagation, the hopping rates are reduced. The resulting desorption patterns are discussed in terms of the jump probabilities and are shown to approach the corresponding analytical solutions in the limiting cases of isotropic and unidirectional diffusions.

(C) 2006 American Institute of Physics. [DOI: 10.1063/1.2161216]
\end{abstract}

\section{INTRODUCTION}

Recent progress in synthesis has given rise to an exponentially increasing variety of nanostructured porous materials. ${ }^{1}$ Hence, the optimization of the pore architecture of such materials with respect to their technical application for, e.g., matter conversion or separation has become a challenging issue of theoretical analysis with an immediate practical background. Examples of such attempts are given in Refs. 2-4. Already two decades ago, stimulated by the first syntheses of the members of the family of MFI-type zeolites ${ }^{5,6}$ the conception of molecular traffic control (MTC) has been suggested ${ }^{7}$ as a first prominent example of such activities. The conception of MTC implies that during catalytic reactions the reactant and product molecules occupy different parts of the channel system with different probabilities. In the extreme case (the so-called "hard" MTC), they may even occupy completely different parts of the pore system. In this way, the reactant molecules on entering and the product molecules on leaving the adsorbent particle may propagate on different pathways, which leads to a minimum of mutual hindrance of the respective diffusion fluxes and hence may lead to a maximum in the product output for transport-controlled processes.

While over essentially two decades the feasibility of this conception has remained a subject of controversial discussion, ${ }^{8-14}$ recent molecular-dynamics simulations with two-component zeolitic adsorbate-adsorbent systems have clearly shown that the prerequisite of MTC, viz., a component-dependent probability distribution within the channel network, may be expected to occur for quite a variety of systems. ${ }^{15}$

${ }^{a)}$ Electronic mail: brzank@uni-leipzig.de
Moreover, considering arrays of mutually intersecting sets of parallel single-file systems ${ }^{16}$ in Ref. 17 for the first time a model system has been established which allowed a quantitative estimation of the possible benefit of MTC for catalytic performance. ${ }^{16}$ As a most simple case, the unidirectional, monomolecular reaction $A \rightarrow B$ was considered, where the reaction was assumed to take place exclusively on intersection sites. In the so-called reference system (REF), the molecular species $A$ and $B$ were accommodated by all channels with equal probability. MTC was implemented in different specifications, expressed by different occupation probabilities of the two molecular species in the two mutually intersecting sets of parallel channels, referred to as $\alpha$ and $\beta$ channels: Under the hard MTC conditions, ${ }^{18-20} A$-type particles are only allowed to enter the $\alpha$ channels, while particles of type $B$ were only accommodated by the $\beta$ channels. Thus, any reaction $A \rightarrow B$ on sites different from intersection sites were excluded, since either the presence of the reactant or of the product molecule in a given channel would be excluded by the hard MTC condition. Therefore, in Ref. 18 also the case of partial release of the hard MTC condition was considered by introducing (finite) probabilities $P_{A, \beta}$ and $P_{B, \alpha}$ that, starting from an intersection, molecules of type $A$ may also enter the $\beta$ channels, and molecules of type $B$ may also enter the $\alpha$ channels, respectively. Theoretical progress has been made in Refs. 9 and 20 where the master equation for the hard MTC system was solved for the very large and very small reactivity case.

On assessing the practical relevance of model calculations with such MTC networks one has to have in mind that in real zeolitic host-guest systems the number of adsorption sites in the channel intersections is comparable with and in some cases even larger than their number in the connecting 

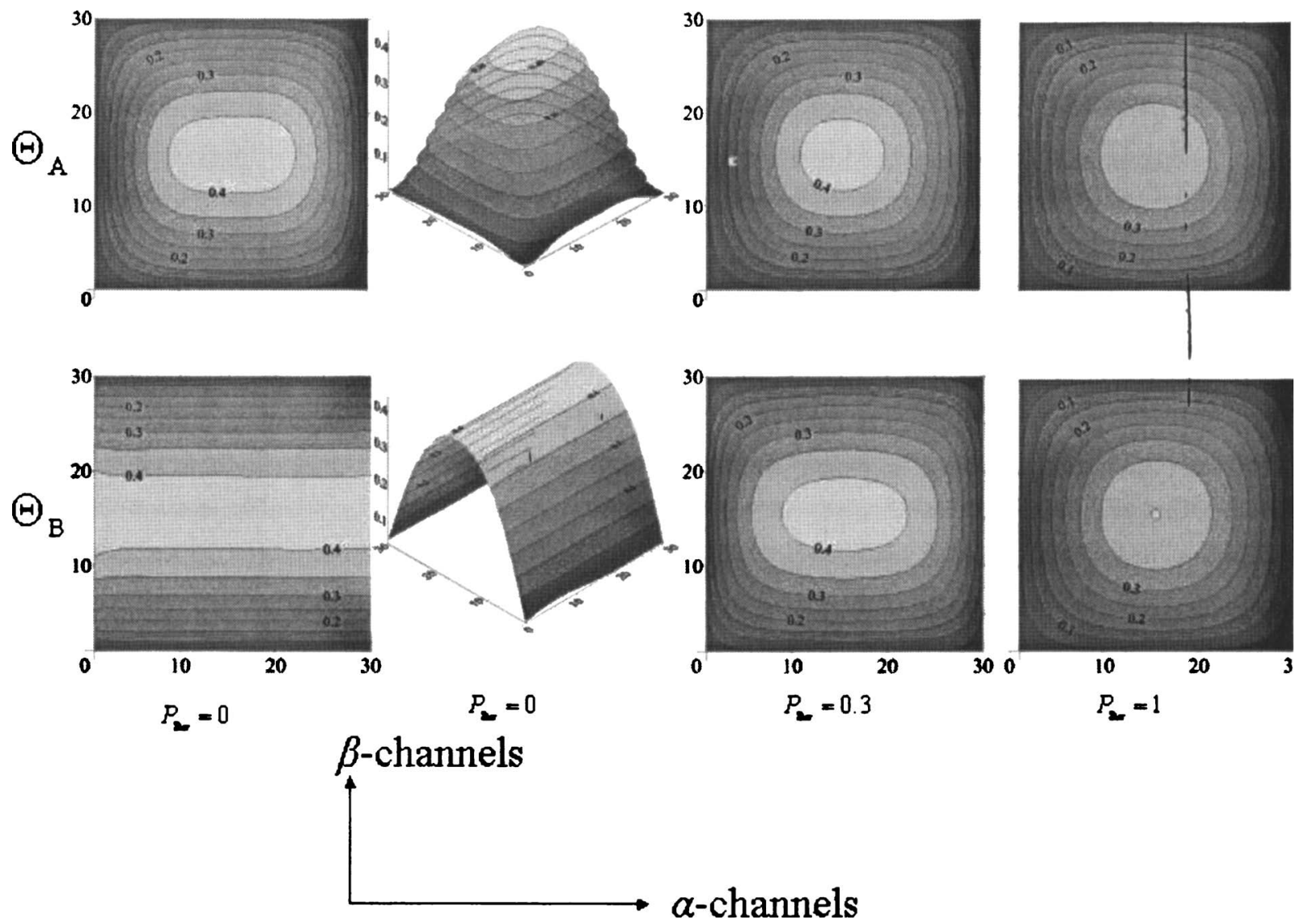

FIG. 1. Concentration profiles of the $A$-type molecules $\Theta_{A}$ (upper line) and $B$-type molecules $\Theta_{B}$ (lower line) after 100 time steps $(t=100 \tau)$ which have been determined under the assumption that the $A$-type molecules have equal accessibility to the $\alpha$ and $\beta$ channels $\left(P_{A \beta}=1\right)$ and that the $B$-type molecules are (i) completely forbidden to enter the $\alpha$ channels $\left(P_{B \alpha}=0\right.$, first column) and are (ii) able to enter with probability $\left(P_{B \alpha}=0.3\right.$, third column) or (iii) enter the $\alpha$ channels with the same probability as the $\beta$ channels $\left(P_{B \alpha}=1\right.$, fourth column). In addition to the isoline representation, in the case of $P_{B \alpha}=0$ also a spatial representation of the concentration profiles is given (second column).

channel elements. Therefore, and for the sake of simplicity, in the present communication we confine ourselves to the limiting case of channel networks with no sites at all between the channel intersections. In this way, molecular trajectories are restricted due to differences in the probability of the jump direction rather than differences in the occupation of different types of channel sites.

\section{SIMULATION MODEL}

We consider a quadratic lattice of $n \times n$ sites, with $n$ $=31$. The separation between adjacent sites shall be denoted by $\lambda$. During the time unit $\tau$ of the simulations $(=1$ Monte Carlo time step), $n^{2}$ sites of the lattice are stochastically addressed. If the selected site is occupied by a particle, one out of the four possible directions towards adjacent sites is stochastically selected to perform a jump attempt with the uniform probability $1 / 4$. Jump attempts are only successful if the site is vacant, to which the attempt is directed. If selected, for both types of particles jump attempts along one direction (i.e., in MTC terms, for $A$ particles into the direction of the $\alpha$ channels and for $B$ particles in the direction of the $\beta$ channels) are unrestrictedly possible. Along the other direction, the probability of successful jump attempts of particles of type $A(B)$ is additionally reduced by the factor $P_{A \beta}\left(P_{B \alpha}\right)$. Jump attempts from the marginal sites are assumed to be performed with the same probabilities as from the internal sites, with the self-evident understanding that jump attempts out of the lattice are not affected by neighboring particles.

Prior to the simulation runs, molecules of types $A$ and $B$ are statistically distributed over the lattice according to the considered initial loading. In all cases considered in this paper the initial loading corresponded to an average site occupancy of $\Theta=0.9$, with equal numbers of $A$ - and $B$-type molecules, i.e., $\Theta_{A}=\Theta_{B}=0.45$. All simulations have been performed in 1000 different runs, using sets of random numbers generated by the algorithm introduced in Ref. 21 . We have used the software package ${ }^{22}$ for a smoothed presentation of the concentration profiles during molecular desorption.

A complete representation of all results, including the raw data and different initial loadings, may be found in Ref. 23.

\section{RESULTS AND DISCUSSION}

\section{A. Presentation and discussion of the attained concentration profiles}

As an example of the concentration profiles resulting from our simulations, Fig. 1 shows the distribution of the $A$-type molecules (upper line) and $B$-type molecules (lower line) after 100 time steps $(t=100 \tau)$ which have been deter- 

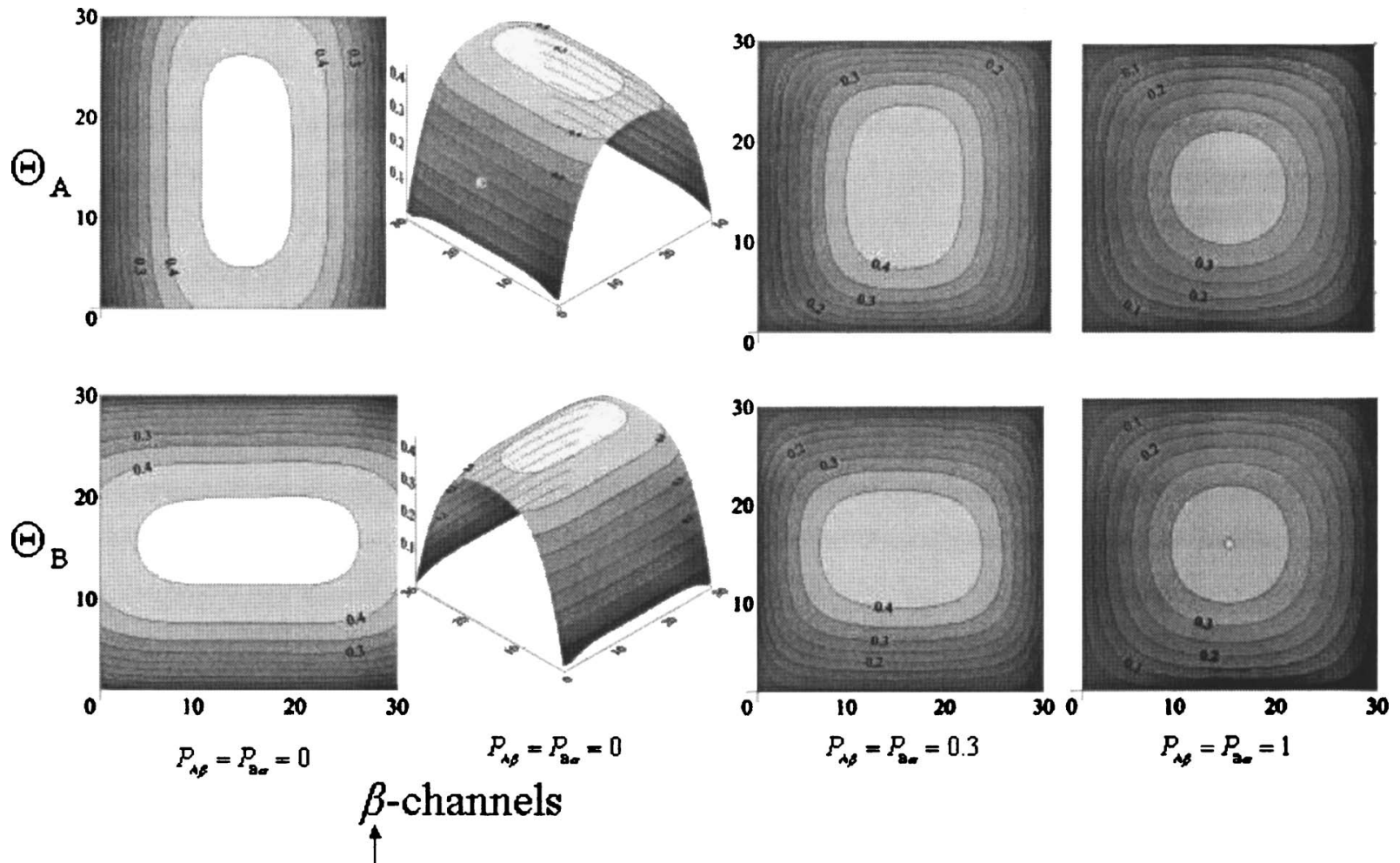

- $\alpha$-channels

FIG. 2. Concentration profiles of the $A$-type molecules $\Theta_{A}$ (upper line) and $B$-type molecules $\Theta_{B}$ (lower line) after 100 time steps $(t=100 \tau)$ for the symmetric case, where the MTC condition for $A$ and $B$ molecules is applied for both types of particles (i.e., $P_{A \beta}=P_{B \alpha}$ ) rather than being totally abandoned for $A$ molecules in all cases. Both the $A$-type molecules and the $B$-type molecules are (i) completely forbidden to enter the other channels $\left(P_{A \beta}=P_{B \alpha}=0\right.$, first column) and are (ii) able to enter with probability $P_{A \beta}=P_{B \alpha}=0.3$ (third column) or (iii) enter the all channels with the same probability $P_{A \beta}=P_{B \alpha}=1$, (fourth column). In addition to the isoline representation, in the case of $P_{A \beta}=P_{B \alpha}=0$ also a spatial representation of the concentration profiles is given (second column).

mined under the assumption that the $A$-type molecules have equal accessibility to the $\alpha$ and $\beta$ channels $\left(P_{A \beta}=1\right)$ and that the $B$-type molecules are (i) completely forbidden to enter the $\alpha$ channels $\left(P_{B \alpha}=0\right.$, left $)$ and are (ii) able to enter with probability $\left(P_{B \alpha}=0.3\right.$, third column) or (iii) enter the $\alpha$ channels with the same probability as the $\beta$ channels $\left(P_{B \alpha}=1\right.$, right). In addition to the isoline representation, in the case of $P_{B \alpha}=0$, for illustration, also a spatial representation of the concentration profiles is given.

The two lines of representations showing the concentration profiles of the $A$ - and $B$-type molecules after 100 simulation steps nicely represent the different situations with the two molecules. While in the chosen example the $A$-type molecules may perform jump attempts in both horizontal direction (the $\alpha$ channels) and vertical direction (the $\beta$ channels), for the $B$ molecules the probability of jump attempts in the horizontal direction ( $\alpha$ channels) decreases from right to left. On the utmost left, this probability is even zero, which appears in the fact that the concentration of molecules $B$ is essentially uniform in the horizontal direction. The decay in the $B$ concentrations follows the only direction into which $B$ transfer is possible, the (vertical) direction of the $\beta$ channels. Interestingly, irrespective of the fact that the probability of jump attempts of the $A$ molecules is identical in both channel types, the distribution of the $A$ molecules is not isotropic (top left). This is obviously an effect of the anisotropic distribution of the $B$ molecules.

Representing the case $P_{A \beta}=P_{B \alpha}=1$, the concentration profiles on the right should reflect total symmetry with respect to both the channels and molecular species. In fact, for both the $A$ and $B$ molecules, the concentration profiles are identical with respect to the directions of the $\alpha$ and $\beta$ channels. Correspondingly, the concentration profiles for the $A$ and $B$ molecules are identical. The appearing slight difference visualizes the effect of the statistical uncertainty in the simulation and the smoothing procedure.

As expected, the profiles in the medium case, $P_{B \alpha}=0.3$, turn out to be intermediate between the respective limiting patterns on the left and on the right. It is worth pointing out that with further increasing time eventually the anisotropy in the profiles is going to vanish, in complete analogy with the cooling behavior of rods with a quadratic cross section. ${ }^{24}$ In the present analysis, however, we are beyond the range where such effects become relevant. ${ }^{25}$

Figure 2 represents the symmetric case, where-in contrast to the situation displayed in Fig. 1-the MTC condition is applied for both types of particles (i.e., $P_{A \beta}=P_{B \alpha}=1$ ) rather than being totally abandoned for $A$ molecules in all cases. As to be required, the symmetry in the requirements 

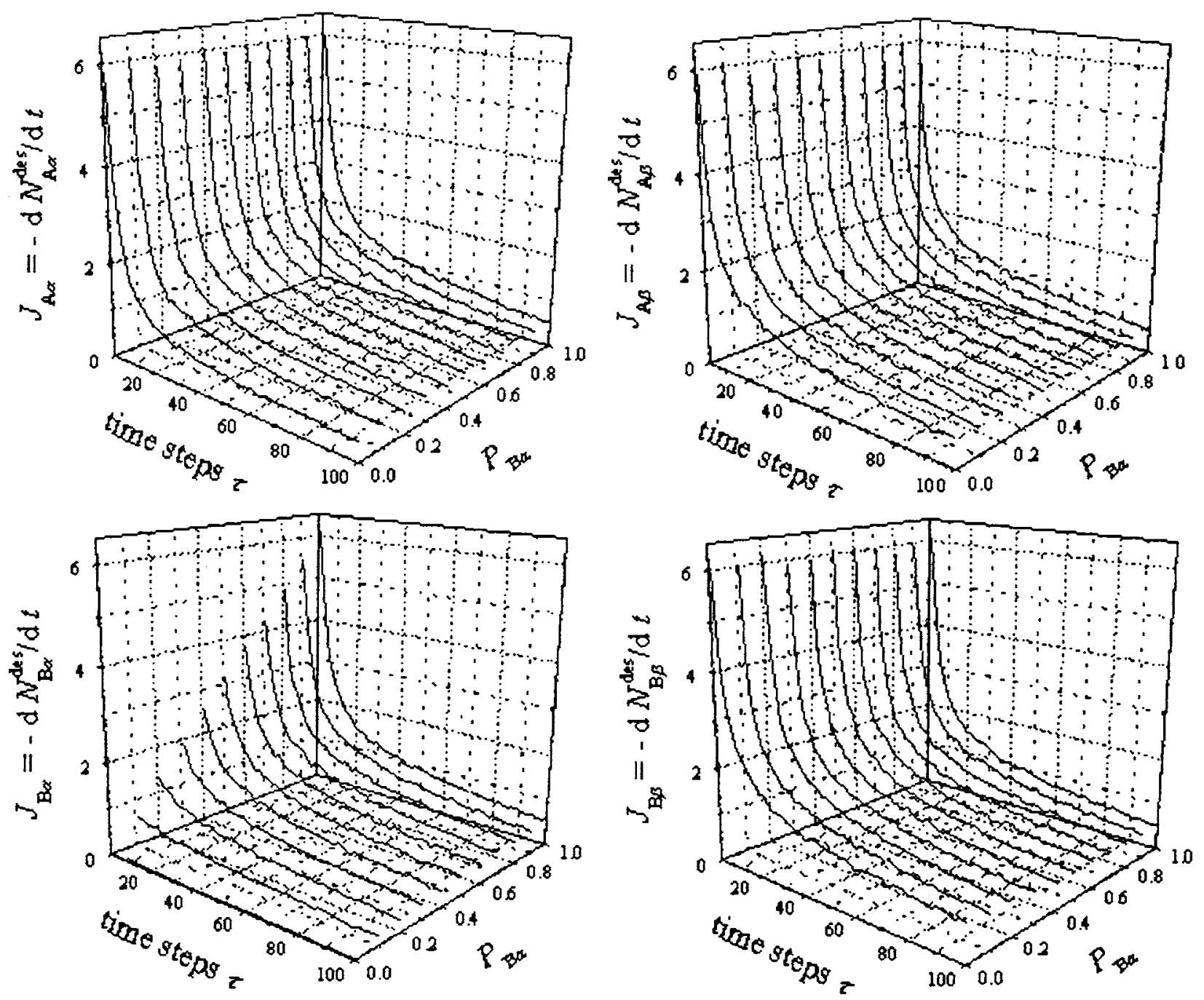

FIG. 3. The time dependence of the four relevant molecular fluxes, viz., of $A$ - and $B$-type molecules out of the $\alpha$ and $\beta$ channels $J_{A \alpha}, J_{A \beta}, J_{B \alpha}$, and $J_{B \beta}$, for $P_{A \beta}=1$ after 100 time steps $(t=100 \tau)$ as a function of the probabilities $0 \leqslant P_{B}^{\alpha} \leqslant 1$ for the jumps of the $B$-type molecules into the $\alpha$ channels.

yields complete symmetry with respect to commutating $A$ and $B$ molecules. Moreover, the asymmetry in each of the representations with respect to $\alpha$ and $\beta$ channels increases from right to left, i.e., with decreasing probabilities $P_{A \beta}$. It also appears from this representation (left part) that in the limiting case of $P_{A \beta}=P_{B \alpha}=0$, the total exclusion of jumps and hence of fluxes of the $A(B)$-type molecules in the vertical (horizontal) direction does not necessarily imply the total absence of concentration gradients of the respective molecules into these directions [as might be concluded from the representation of Fig. 1 (bottom, left)]. In fact, such gradients do result as a consequence of gradients in, e.g., the vertical direction of the flux of the $A$-type molecules in the horizontal direction which are clearly not excluded.

Since the concentration profiles as presented in Figs. 1 and 2 contain the whole information resulting from our simulation there is no better way of recording the different states passed by the host-guest systems under study during molecular desorption. In many cases, however, more detailed knowledge of the dependence of certain features of the desorption process on the system parameters is required. Therefore, Sec. III B is devoted to the presentation of some examples of the total molecular flux out of the system and its time integral, i.e., the total amount of molecules desorbed as a function of the time elapsed after the start of desorption and of the intrinsic probabilities for jump attempts into the direction of the $\alpha$ and $\beta$ channels.

\section{B. Fluxes and amounts desorbed as a function of the intrinsic jump probabilities}

Figure 3 shows the time dependence of the four relevant molecular fluxes, viz., of $A$ - and $B$-type molecules out of the $\alpha$ and $\beta$ channels $\left(J_{A \alpha}, J_{A \beta}, J_{B \alpha}\right.$, and $\left.J_{B \beta}\right)$, respectively. The notations at the vertical coordinates indicate that the fluxes result as the (negative) derivatives of the respective total amounts desorbed $\left(N_{A \alpha}^{\mathrm{des}}, N_{A \beta}^{\mathrm{des}}, N_{B \alpha}^{\mathrm{des}}\right.$, and $\left.N_{B \beta}^{\mathrm{des}}\right)$, such as, vice versa, the total amounts desorbed follow from the time integrals over the fluxes. The presentations of Fig. 3 correspond to Fig. 1 where the $A$-type molecules have also been assumed to jump unrestrictedly into the direction of the $\alpha$ and $\beta$ channels. However, the fluxes shown in Fig. 3 refer not only to the instant of time for which the profiles have been presented (100 simulation time steps after onset of desorption) but also include the whole time dependence up to this point. Moreover, the probability $P_{B \alpha}$ that a $B$ molecule performs a jump 

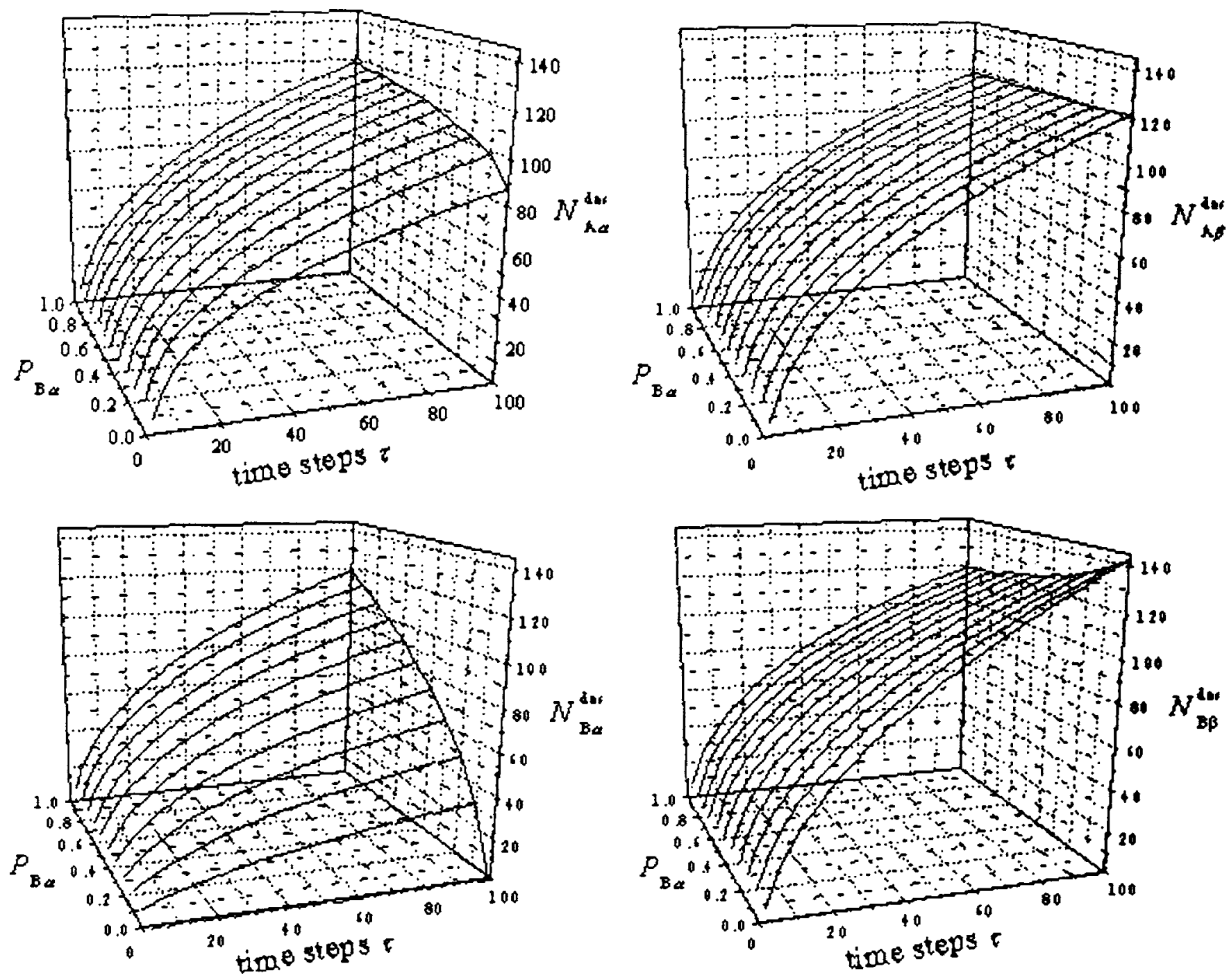

FIG. 4. The total amounts desorbed $N_{A \alpha}^{\mathrm{des}}, N_{A \beta}^{\mathrm{des}}, N_{B \alpha}^{\mathrm{des}}$, and $N_{B \beta}^{\mathrm{des}}$ as a function of the number of time steps elapsed after the onset of desorption for the same conditions, as considered in Fig. 3.

attempt into an $\alpha$ channel is varied over the entire possible range, rather than choosing only the values $0,0.3$, and 1 , like in Fig. 1. Note that the fluxes of the molecules $A$ out of the $\alpha$ and $\beta$ channels and of the molecules $B$ out of the $\beta$ channels appear to be uniform and keep essentially unaffected with a variation of $P_{B \alpha}$. This is not unexpected since a variation of $P_{B \alpha}$ only concerns the flux rates of $B$ molecules in the $\alpha$ direction. The latter correlation is nicely reflected by the corresponding representation of Fig. 3 (bottom left), where the initial flux rates for the $B$ molecules out of the $\alpha$ channels are seen to increase in proportion to $P_{B \alpha}$, including the patterns expected for the limiting cases, i.e., no flux at all for $P_{B \alpha}$ $=0$ and a pattern essentially coinciding with those of the presentations (top and bottom right) of $J_{A \alpha}, J_{A \beta}$, and $J_{B \beta}$, for $P_{B \alpha}=1$.

In view of the similarity of the trends of $J_{A \alpha}, J_{A \beta}$, and $J_{B \beta}$, a more detailed investigation of the influence of the intrinsic jump probabilities should be based on a discussion of the total amounts desorbed, i.e., on the time integrals of the respective fluxes rather than on the fluxes themselves. In this way existing, but rather faint, differences may become more easily observable. For this purpose, Fig. 4 displays the total amounts desorbed as a function of the number of time steps elapsed after the onset of desorption for the same conditions, as considered in Fig. 3. In fact, Fig. 4 exhibits nothing else than the time integrals over the corresponding functions shown in Fig. 3, plotted as a function of the upper limits of the integrals.

Again, the time dependence of the total amount of $B$ molecules desorbed from the $\alpha$-channels $\left(N_{B \alpha}^{\text {des }}\right.$, bottom left) behaves notably different from the other dependencies. In contrast to the initial part (note that Fig. 3 has shown the respective slopes), however, with increasing simulation time the increase in the total amount desorbed in the $\alpha$ direction is increasing less than linearly with the probability $P_{B \alpha}$ for jump attempts of $B$ molecules in the $\alpha$ direction. This may be attributed to the fact that the total amount of $B$ molecules on marginal sites available for desorption decreases with increasing $P_{B \alpha}$, while it is uniform at the onset of desorption.

Now, with the data shown in Fig. 4, also differences in the influence of the probability $P_{B \alpha}$ for jump attempts of $B$ molecules in the $\alpha$ direction on the remaining types of desorption behavior, represented by the time dependence of the quantities $N_{A \alpha}^{\mathrm{des}}, N_{A \beta}^{\mathrm{des}}$, and $N_{B \beta}^{\mathrm{des}}$, become detectable. It is inter- 

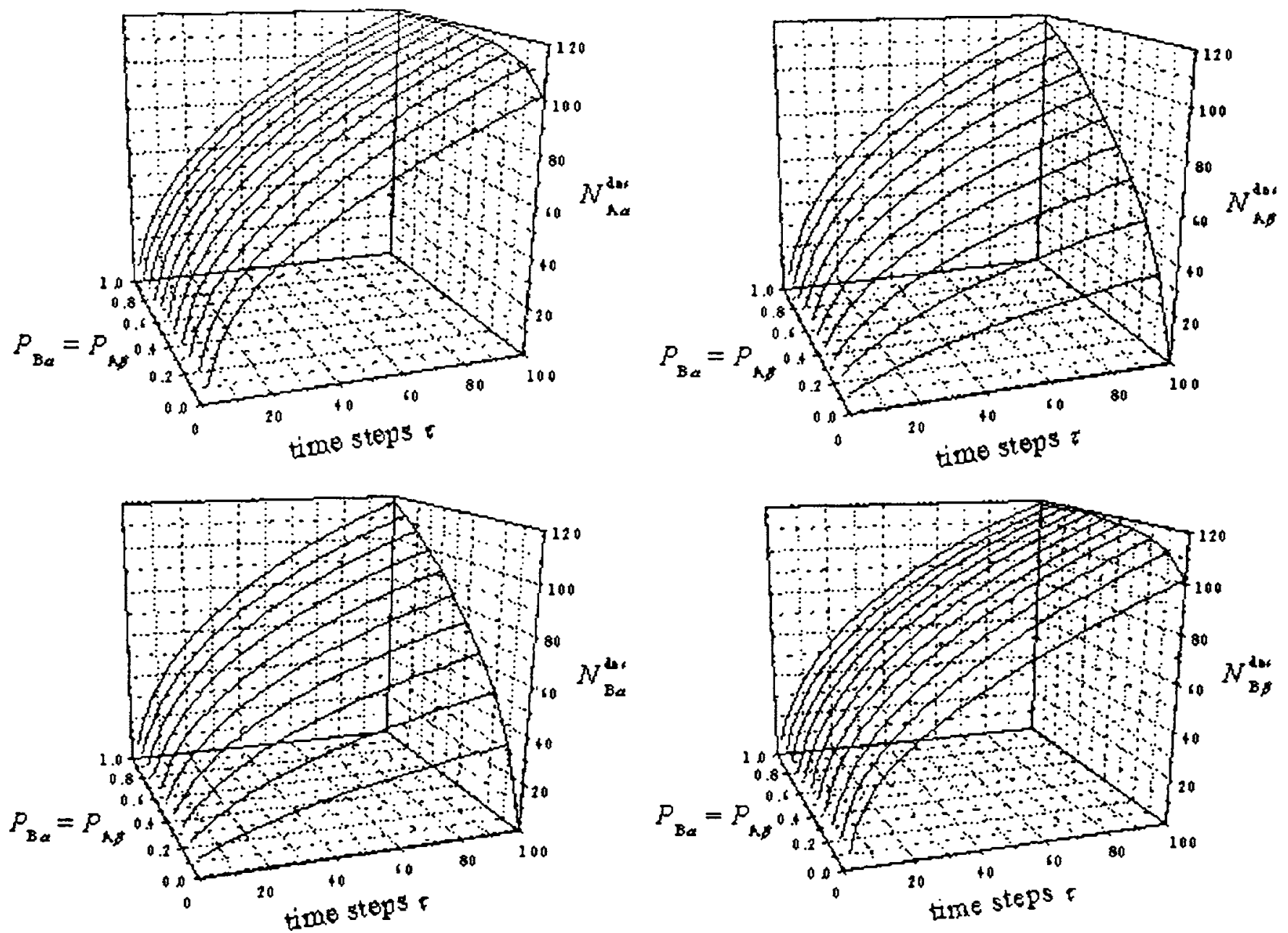

FIG. 5. The total amounts desorbed, $N_{A \alpha}^{\mathrm{des}}, N_{A \beta}^{\mathrm{des}}, N_{B \alpha}^{\mathrm{des}}$, and $N_{B \beta}^{\mathrm{des}}$, as a function of the number of time steps elapsed after the onset of desorption as a function of the probabilities $P_{A \beta}=P_{B \alpha}$.

esting to note that, starting from $P_{B \alpha}=1$ (which has been referred to as the conditions for the reference system REF), all three primary options are observed: the amount of $A$ molecules leaving in the $\beta$ direction $\left(N_{A \beta}^{\text {des }}\right)$ remains unaffected, while their flux in the $\alpha$ direction $\left(N_{A \alpha}^{\mathrm{des}}\right)$ is decreasing and the flux of $B$ molecules in the $\beta$ direction $\left(N_{B \beta}^{\text {des }}\right)$ is increasing. The increase of the amount of $B$ molecules desorbed in the $\beta$ direction with decreasing values of $P_{B \alpha}$ may be understood as a consequence of the reduced escape probability of $B$ molecules in the $\alpha$ direction. The fact that the amount of $A$ molecules desorbed in the $\alpha$ direction increases with decreasing values of $P_{B \alpha}$ may intuitively be attributed to some push-pull effect by the increased desorption of $B$ molecules into the same direction.

Figure 5 displays the total amounts desorbed under the condition of symmetry with respect to the channels and molecules, as illustrated for a few selected examples by the concentration profiles given in Fig. 2. As to be required, this symmetry is completely reflected by the representations, showing coinciding dependencies for $N_{A \alpha}^{\mathrm{des}}$ and $N_{B \beta}^{\mathrm{des}}$ as well as for $N_{A \beta}^{\mathrm{des}}$ and $N_{B \alpha}^{\mathrm{des}}$ as a function of $P_{A \beta}=P_{B \alpha}$. Again, the amount of $A(B)$ molecules desorbed in the "other" [viz., the $\beta(\alpha)$ ] direction notably decreases with decreasing values $P_{A \beta}=P_{B \alpha}$, while there is a much fainter ("second-order") ef- fect with the same tendency concerning the amount of molecules desorbed in "their" direction, i.e., $A$ molecules in the $\alpha$ direction and $B$ molecules in the $\beta$ direction.

\section{Moments' analysis and comparison with analytical solutions}

An analysis of uptake/release experiments has been notably facilitated after the so-called moments' analysis, having been well established already in gas chromatography, ${ }^{26}$ was introduced into the field of molecular sorption. ${ }^{27,28}$ The benefit of this approach results from the fact that the information contained in the time dependence of an adsorption/ desorption curve is referred to a series of quantities for which, depending on the given limiting mechanism, simple analytical expressions may be derived. In the present context it is sufficient to confine to the so-called first moment $M_{1}$. It is defined by the equation

$$
M_{1}=\int_{0}^{\infty}\left[1-\frac{N^{\operatorname{des}}(t)}{N^{\operatorname{des}}(\infty)}\right] d t .
$$




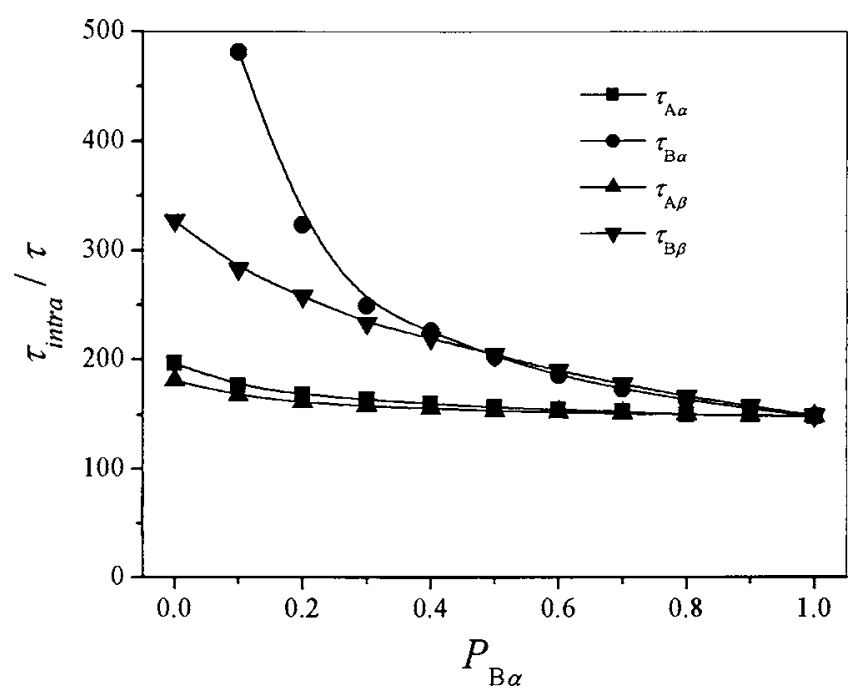

FIG. 6. Intracrystalline mean lifetimes $\tau_{\text {intra }}$ (in units of the time step duration $\tau$ ) determined for the desorption processes shown in Fig. 4 for the first 100 time steps as a function of the probabilities $P_{B \alpha}$ for the case $P_{A \beta}=1$.

$N^{\operatorname{des}}(t)$ is the total amount desorbed at time $t$. We shall consider the quantities $N_{A \alpha}^{\mathrm{des}}, N_{A \beta}^{\mathrm{des}}, N_{B \alpha}^{\mathrm{des}}$, and $N_{B \beta}^{\mathrm{des}}$, whose time dependences over the first 100 time steps of the simulations are presented in Figs. 4 and 5. The first moment is nothing else than the medium time a molecule, contributing to a certain outflow, remains within the system after the onset of the desorption process. Therefore, it is generally referred to as the intracrystalline mean lifetime of the molecular species under consideration.

Figure 6 displays the intracrystalline mean lifetimes $\tau_{\text {intra }}$ (in units of the time step duration $\tau$ ) determined for the desorption processes shown in Fig. 4 for the first 100 time steps. The obtained data are in agreement with the expected behavior. In all cases, the intracrystalline mean lifetimes decrease with decreasing internal confinement, i.e., with increasing probability that the $B$ molecules are permitted to perform jump attempts also in the $\alpha$ direction. Eventually, for $P_{B \alpha}=1$, there is no difference anymore in the molecules and channels. Correspondingly, all dependencies merge in one point.

With the exception of this limiting point, the intracrystalline lifetimes of the $A$-type molecules are notably shorter than those of the $B$ molecules. This corresponds to the fact that in the considered case the $B$ molecules are subject to an additional restriction in comparison with the $A$ molecules. This restriction, in particular, concerns the flux of $B$ molecules out of the system in the $\alpha$ direction. In view of Fig. 4 , exhibiting essentially identical curves for the quantities $N_{A \alpha}^{\mathrm{des}}, N_{A \beta}^{\mathrm{des}}$, and $N_{B \beta}^{\mathrm{des}}$, at a first glance one may be astonished about the notably longer intracrystalline mean lifetime $\tau_{B \beta}$ in comparison to $\tau_{A \alpha}$ and $\tau_{A \beta}$, attaining a factor of nearly 2 for $P_{B \alpha}=0$. This, however, is a consequence of the fact that at the onset of desorption our channel system hosts as many $A$

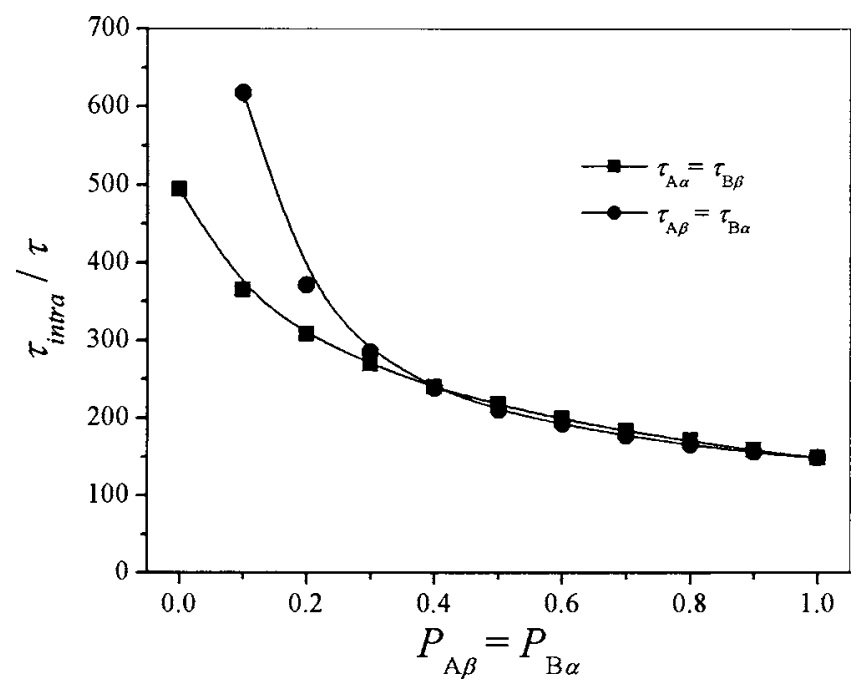

FIG. 7. Intracrystalline mean lifetimes in the case $P_{A \beta}=P_{B \alpha}$, which corresponds to the situations shown in Fig. 5.

as $B$ particles. Hence, it may be intuitively accepted that the mean lifetime of the $A$ molecules, which may use both types of "exits," should have a correspondingly shorter intracrystalline mean lifetime than the $B$ molecules, which may unrestrictedly leave the system only in the $\beta$ direction.

Figure 7 shows the intracrystalline mean lifetimes in the case $P_{A \beta}=P_{B \alpha}$, which corresponds to the situations shown in Figs. 2 and 5. Due to symmetry, there are only two different dependencies to be considered, viz., (i) for the molecules leaving through "their" channels, i.e., $A$ through $\alpha$ and $B$ through $\beta$, and (ii) for the molecules leaving through "the other" channels. Starting from a joint value at $P_{A \beta}=P_{B \alpha}=1$ (the same as in Fig. 6), the mean lifetimes increase with increasing confinement (i.e., with decreasing jump probabilities $P_{A \beta}=P_{B \alpha}$ ). Moreover, the intracrystalline mean lifetimes for molecules leaving through the other channels become increasingly larger than those for the molecules leaving through their channels.

Molecular diffusions in single-file channels and in channel networks of guest-dependent anisotropy ${ }^{29}$ known as highly correlated systems, so far accessible to analytic approaches only in some limiting cases. In the system considered in this communication, such a limiting case is expected to exist for $P_{A \beta}=P_{B \alpha}=1$. Under these conditions, the distinction between the molecules and the channels has vanished and the results of our simulations should reflect the process of diffusion-limited desorption from a square.

In Ref. 28, the analytical expression of the intracrystalline mean lifetime within a parallelepiped of extensions $2 a, 2 b, 2 c$ may be found to be

$$
\tau_{\text {intra }}=\frac{4}{D \pi^{2}}\left(\frac{8}{\pi^{2}}\right)^{3} \sum_{i=0}^{\infty} \sum_{j=0}^{\infty} \sum_{k=0}^{\infty} A(i, j, k, a, b, c),
$$

with

$$
A=\frac{1}{(2 i+1)^{2}(2 j+1)^{2}(2 k+1)^{2}\left\{[(2 i+1) / a]^{2}+[(2 j+1) / b]^{2}+[(2 k+1) / c]^{2}\right\}}
$$


The relevant conditions for the desorption from a square of edge length $2 a$ into the surrounding plane, here to be considered, coinciding with those for desorption from an infinitely long rod of quadratic cross section into the surrounding space. Therefore one has $a=b$ und $c \rightarrow \infty$, and $A$ simplifies to $A(i, j, k, a)=\frac{a^{2}}{(2 i+1)^{2}(2 j+1)^{2}(2 k+1)^{2}\left[(2 i+1)^{2}+(2 j+1)^{2}\right]}$.

With Eq. (4), the desired analytical expression of the intracrystalline mean lifetime assumes the form

$$
\tau_{\text {intra }}=\frac{4}{D \pi^{2}}\left(\frac{8}{\pi^{2}}\right)^{3} a^{2} S_{1},
$$

with

$$
\begin{aligned}
S_{1}= & \sum_{i=0}^{\infty} \sum_{j=0}^{\infty} \frac{1}{(2 i+1)^{2}(2 j+1)^{2}\left[(2 i+1)^{2}+(2 j+1)^{2}\right]} \\
& \times \sum_{k=0}^{\infty} \frac{1}{(2 k+1)^{2}} .
\end{aligned}
$$

Using the analytical expression ${ }^{30}$

$$
\sum_{k=0}^{\infty} \frac{1}{(2 k+1)^{2}}=\sum_{k=1}^{\infty} \frac{1}{(2 k-1)^{2}}=\frac{\pi^{2}}{8} .
$$

For the second term on the right-hand side of Eq. (6), and a value of 0.528 for the double sum as resulting from numerical calculation, the intracrystalline mean lifetime is found to be given by the expression

$$
\tau_{\text {intra }} / \tau=\frac{n^{2}}{7.11357},
$$

where we have made use of the relation

$$
D=\frac{\lambda^{2}}{4 \tau}
$$

for transport diffusion of hard-core particles on a quadratic lattice, ${ }^{31}$ coinciding with the self-diffusivity at infinite dilution. $^{32}$ Moreover, for deriving Eq. (8) the edge length of our model host-guest system corresponding to the value of $2 a$ of the analytical expression has been assumed to be equal to $n \lambda$. Note that the distance between the corner sites is only $(n-1) \lambda$. However, in the solution of the diffusion equation leading to the analytical expression of the intracrystalline mean lifetime, ${ }^{33,34}$ the relevant boundary conditions require the total absence of molecules at the margins (vanishing sorbate concentration), while within our network also marginal sites may be occupied. Zero concentration may therefore only be assumed to be realized outside of the array, which is taken into account by the slightly enhanced value of its extension $[n \lambda$, rather than $(n-1) \lambda]$ corresponding to the extension $2 a$ of the analytically treated system. With an increasing array size, the significance of this difference is clearly going to vanish.

Inserting, as considered in our example, $n=31$ into Eq. (8) yields a value of $\tau_{\text {intra }}=135.1 \tau$, which is, in fact, in satis- factory agreement with the result of our simulations in the limiting case $P_{A \beta}=P_{B \alpha}=1$ (last point on the right of Figs. 6 and 7), where a value of $\tau_{\text {intra }}=147 \tau$ was obtained. The theory is set up in continuous space and ignore correlation between particles which explains the deviation of about $8 \%$. The observed, slightly larger intracrystalline mean lifetime is reasonable since particles in the bulk of the system are captured by those located closer to the surface.

For a parallel-sided slab of thickness $2 a$, the intracrystalline mean lifetime may be found ${ }^{28}$ by the expression

$$
\tau_{\text {intra }}=\frac{a^{2}}{3 D} \text {. }
$$

This relation might be compared with the results of our simulations when molecular transport is possible in one direction only. For adapting Eq. (10) to the situation relevant for our simulations, again $2 a$, the effective file length, is put equal to $n \lambda$. Also for expressing the diffusivity, we have again to apply Eq. (9). At a first glance, this seems to be in contrast with the well-known relation ${ }^{35}$

$$
D=\frac{\lambda^{2}}{2 \tau_{\text {jump }}},
$$

where $\tau_{\text {jump }}$ is the mean time between two jump attempts in file direction. For the $B$ molecules under study, however, the mean time between two jumps in the file direction is equal to $2 \tau$, since each second jump attempt will be directed perpendicular to this direction and shall be in vain due to the condition $P_{B \alpha}=0$. Thus Eqs. (9) and (11) are identical.

With this relation inserted, Eq. (10) becomes

$$
\tau_{\text {intra }}=\frac{n^{2}}{3} \tau \text {, }
$$

yielding a value of $\tau_{\text {intra }}=320.3 \tau$ for the case considered. Again, a reasonable agreement with the value of $\tau_{B \beta}$ $=327.3 \tau$ (Fig. 6) obtained in our simulations for $P_{A \beta}=1$ and $P_{B \alpha}=0$ is observed. This agreement is particularly remarkable in view of an obvious difference in the analytical treatment leading to Eq. (10) and our simulations. While Eq. (10) is derived for one-component diffusion, in our simulations molecular diffusion of the $B$ molecules has to proceed under the presence of the $A$ molecules. However, since their mobility in the $\beta$ direction coincides with that of the $B$ molecules, obviously their presence does in no way affect the desorption dynamics of the $B$ molecules.

\section{CONCLUSIONS}

Molecular desorption of a two-component molecular mixture from a pore system exhibiting different interaction patterns with the guest molecules is studied by dynamic Monte Carlo simulations. The situation considered is typical for nanoporous catalysts with potentials for reactivity enhancement by molecular traffic control, i.e., of systems where the molecules involved (viz., reactants and products) are directed along different pathways. In the present study we have confined ourselves to two-component desorption, where the direction of preferred desorption for the components is perpendicular to each other and where the probabil- 
ity of migration perpendicular to this direction is varied between zero and the value for propagation in the preferential direction.

The consistency of the obtained results has been illustrated by heuristic arguments and confirmed by their agreement with analytical approaches in the limiting cases of (i) isotropic diffusion of both components and of (ii) onedimensional diffusion of one component and isotropic diffusion of the other.

With the advent of imaging techniques such as interference microscopy $^{36}$ and IR microscopy, ${ }^{37}$ two-component concentration patterns such as those shown in Figs. 1 and 2 as well as the related quantities may become accessible to direct experimental observation. As a first step into this direction, in Ref. 38 the concentration profiles during methanol desorption from zeolite crystals of type ferrierite are presented. Exhibiting a system of two arrays of parallel channels with different diameters, mutually intersecting each other, ${ }^{1}$ zeolites of this type are ideally suited as a host system serving as the experimental counterpart for simulations of the presented type.

It is noteworthy that zeolite crystals of the type ferrierite are traversed by two-dimensional channel systems which are ideally suited to a comparison between the present theoretical treatment and direct experimental observation in hopefully near future. The presented problem, however, is clearly of more general relevance. Similarly as in the considered case of two-dimensional pore networks, adsorbate-related diffusion anisotropy is also expected to occur in zeolites with genuine three-dimensional pore architecture. Remarkably, one of the most important family of zeolites for heterogenous catalysis, $^{39}$ the MFI-type zeolites (with ZSM-5 and silicalite-1 as their most important representatives) consists of two types of channels, with the straight and sinusoidel ones. As a consequence of their special arrangement, the alternating motion in elements of these two channel systems gives rise to diffusion in the third dimension. ${ }^{40,41}$ Hence, in some way, this type of zeolites might be considered as a transition case between the two-dimensional case considered in this study and the genuine three-dimensional diffusion.

\section{ACKNOWLEDGMENTS}

Financial supports by the Deutsche Forschungsgemeinschaft (Ka. 953/15 and International Research Training Group "Diffusion in Zeolites") and by Fonds Chemischer Industrie are gratefully acknowledged.

\footnotetext{
${ }^{1}$ Ch. Baerlocher, W. H. Meier, and D. H. Olson, Atlas of Zeolite Structure Types (Elsevier, London, 2001).

${ }^{2}$ D. S. Sholl and K. A. Fichthorn, J. Chem. Phys. 107, 4384 (1997).

${ }^{3}$ P. Adhangale and D. Keffer, Sep. Sci. Technol. 38, 977 (2003).
}

${ }^{4}$ K. F. Czaplewski, T. L. Reitz, Y. J. Kim, and R. Q. Snurr, Microporous Mesoporous Mater. 56, 55 (2002).

${ }^{5}$ G. T. Kokotailo, S. L. Lawton, D. H. Olson, and W. M. Meier, Nature (London) 272, 437 (1978).

${ }^{6}$ E. M. Flanigen, J. M. Bennett, R. W. Grose, J. P. Cohen, R. L. Patton, R. M. Kirchner, and J. V. Smith, Nature (London) 271, 512 (1978).

${ }^{7}$ E. G. Derouane and Z. I. Gabelica, Catalysis 65, 486 (1980).

${ }^{8}$ B. M. Lowe, D. A. Whan, and M. S. Spencer, J. Catal. 70, 237 (1981).

${ }^{9}$ E. G. Derouane, Z. Gabelica, and P. A. Jacobs, J. Catal. 70, 238 (1981).

${ }^{10}$ C. G. Pobe, J. Catal. 72, 174 (1981).

${ }^{11}$ E. G. Derouane, J. Catal. 72, 177 (1981).

${ }^{12}$ E. G. Derouane, Appl. Catal., A 115, N2 (1994).

${ }^{13}$ F. Keil Diffusion und Chemische Reaktion in der Gas/Feststoff-Katalyse (Springe, Berlin, 1999).

${ }^{14}$ J. Weitkamp and L. Puppe, Catalysis and Zeolites (Springer, Berlin, 1999).

${ }^{15}$ L. A. Clark, G. T. Ye, and R. Q. Snurr, Phys. Rev. Lett. 84, 2893 (2000).

${ }^{16}$ N. Neugebauer, P. Bräuer, and J. Kärger, J. Catal. 194, 1 (2000).

${ }^{17}$ P. Bräuer, J. Kärger, and N. Neugebauer, Europhys. Lett. 53, 8 (2001).

${ }^{18}$ P. Bräuer, A. Brzank, and J. Kärger, J. Phys. Chem. 107, 1821 (2003).

${ }^{19}$ A. Brzank, G. M. Schütz, P. Bräuer, and J. Kärger, Phys. Rev. E 69, 031102 (2004).

${ }^{20}$ A. Brzank and G. M. Schütz, Appl. Catal., A 288, 194 (2005).

${ }^{21}$ G. Marsaglia and A. Zaman, Stat. Probab. Lett. 9.1, 35 (1990).

${ }^{22}$ SURFER(WIN 32), Version 6.04, Feb 24 1997, Surface mapping system, Golden Software, Inc., 1993.

${ }^{23}$ P. Bräuer, A. Brzank, and J. Kärger (unpublished).

${ }^{24}$ H. Tautz, Wärmeleitung und Temperaturaustausch (Akademie-Verlag, Berlin, 1971).

${ }^{25}$ P. Bräuer, A. Brzank, and J. Kärger (unpublished).

${ }^{26}$ C. N. Satterfield, Mass Transfer in Heterogeneous Catalysis (MIT, Cambridge, 1970).

${ }^{27}$ A. J. Zikanova, J. Chromatogr. Sci. 9, 248 (1971).

${ }^{28}$ R. M. Barrer, Zeolites and Clay Minerals as Sorbents and Molecular Sieves (Academic, London, 1978), p. 272.

${ }^{29} \mathrm{~K}$. W. Kehr, K. Mussawisade, and T. Wichmann in Diffusion in Condensed Matter, edited by J. Kärger, P. Heitjans, and R. Haberlandt (Vieweg, Braunschweig Springer, Berlin, 1998), p. 265-304.

${ }^{30}$ G. A. Korn and T. M. Korn, Mathematical Handbook for Scientists and Engineers, Definitions, Theorems and Formulas for Reference and Review (McGraw-Hill, New York, 1961).

${ }^{31}$ R. Kutner, Phys. Lett. 81A, 239 (1981)

${ }^{32}$ J. Kärger and D. V. Ruthven, Diffusion in Zeolites and Other Microporous Solids (Wiley, New York, 1992).

${ }^{33}$ J. Crank, The Mathematics of Diffusion (Clarendon, Oxford, 1975).

${ }^{34}$ H. S. Carslaw and J. C. Jaeger, Conduction of Heat in Solids (Oxford, Oxford, 2004)

${ }^{35} \mathrm{H}$. Mehrer and F. Wenwer, Diffusion in Condensed Matter, edited by J. Kärger, P. Heitjans, and R. Haberlandt (Vieweg, Braunschweig Springer, Berlin, 1998). p. 1.

${ }^{36}$ E. Lehmann, S. Vasenkov, J. Kärger, G. Zadrozna and J. Kornatowski J. Chem. Phys. 118, 6129 (2003).

${ }^{37}$ M. Hermann, W. Niessen, and H. G. Karge, Catalysis by Microporous Materials, edited by H. K. Beyer, H. G. Karge, I. Kiricsi, and J. B. Nagy (Elsevier, Amsterdam, 1995), p. 131-138.

${ }^{38}$ P. Kortunov C. Chmelik, J. Kärger, R. A. Rakoczy, D. M. Ruthven, Y. Traa, S. Vasenkov, and J. Weitkamp, Adsorption (in press).

${ }^{39}$ J. Weitkamp and L. Puppe, Catalysis and Zeolites (Springer, Berlin, 1999).

${ }^{40}$ J. Kärger, J. Phys. Chem. 95, 5558 (1991).

${ }^{41}$ F. Jousse, S. M. Auerbach and D. P. Vercauteren, J. Chem. Phys. 112, $1531(2000)$ 